\title{
Synthesis, Structure, and Extraction Ability of Tetrasubstituted Thiacalix[4]arenes with Crown Ether Fragments on the Lower Rim
}

\author{
Svetlana E. Solovieva, ${ }^{a}{ }^{@}$ Anton A. Muravev, ${ }^{a}$ Rinat T. Zakirzyanov, ${ }^{\text {b }}$ \\ Shamil' K. Latypov, ${ }^{a}$ Igor' S. Antipin, $^{\mathrm{a}, \mathrm{b}}$ and Alexander I. Konovalov ${ }^{\mathrm{a}}$ \\ ${ }^{a}$ Arbuzov Instute of Organic and Physical Chemistry, Kazan Scientific Center, Russian Academy of Sciences, \\ 420088 Kazan, Russia \\ ${ }^{\mathrm{b} B u t l e r o v}$ Chemical Institute, Kazan [Volga Region] Federal University, 420008 Kazan, Russia \\ ${ }^{\circledR}$ Corresponding author E-mail: svsol@iopc.ru
}

Synthesis of $\omega$-bromoalkoxy derivatives of thiacalix-monocrown ethers in 1,3-alternate conformation was performed. The complexation ability of synthesized macrocycles towards alkali metal cations was investigated by two-phase extraction method.

Keywords: Thiacalix[4]monocrown, picrate extraction, 2D NMR, Mitsunobu reaction.

\section{Introduction}

Recently, the design of high-performance and selective ligands for the metal cations binding, which combine receptor and signal function, attracts the considerable interest of researchers; that can be valuable for the production of sensors, ion-selective electrodes, and so on. One of the promising classes of compounds with these properties are calix[4]arenes 1a and 2a with crown ether fragments (Figure 1).

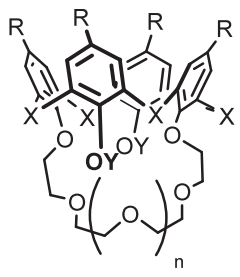

1a cone, 1,3-alternate $1 \mathbf{b}(\mathrm{X}=\mathrm{S})$

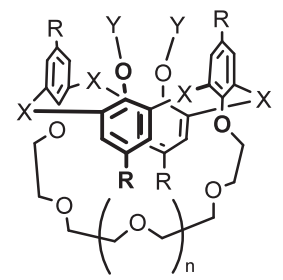

$\left(\mathrm{X}=\mathrm{CH}_{2}\right)$

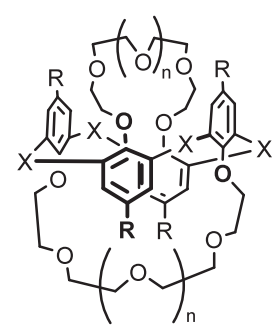

2a $\left(\mathrm{X}=\mathrm{CH}_{2}\right)$ 2b $(\mathrm{X}=\mathrm{S})$
Figure 1. Mono- and bis-(thia)calix[4]crown ethers.

These compounds have the receptor ability towards alkali and alkaline-earth metal cations and their binding selectivity mainly depends on the number of oxygen atoms in polyethylene glycol chain and calixarene stereoisomeric form. ${ }^{[1]}$ Among them very selective receptor molecules were discovered. For example, calix[4]crown-6-ether in 1,3-alternate conformation demonstrated high $\mathrm{Cs}^{+} / \mathrm{Na}^{+}$ selectivity (more than 30000). ${ }^{[2-4]}$

Substitution of methylene bridges for sulfur atoms results in the macrocycle size increase and the formation of new receptor properties for thiacalixarene derivatives. [4-13] Potentiometric sensors based on thiacalix[4]crown ethers $\mathbf{2 b}$ in poly(vinyl chloride) membrane electrodes have the detection limit of cesium cations less than $10^{-7}$ $\mathrm{M} ;{ }^{[16]}$ compounds $\mathbf{1 b}$ with pyridylmethyl substituents in 1,3-alternate conformation ${ }^{[17]}$ demonstrate the ability to switchable recognition of lithium and silver cations; $\mathbf{1 b}$ with methoxy and propoxy groups extract selectively potassium and rubidium cations. ${ }^{[18]}$ Size of thiacalixarene platform is the key parameter ${ }^{[14]}$ to control the complexation ability of derivatives $\mathbf{2} \mathbf{b}$ towards alkali metal ions. The size of crown ether chain plays an important role as well. ${ }^{[15]}$

To use the thiacalixcrowns for sensor design the presence of appropriate terminal functional groups, which can effectively interact with solid surface, is required (Figure 2). For this reason, regio- and stereoselective synthesis of thiacalix-monocrowns derivatives containing lateral omegafunctionalized substituents represents an important problem.

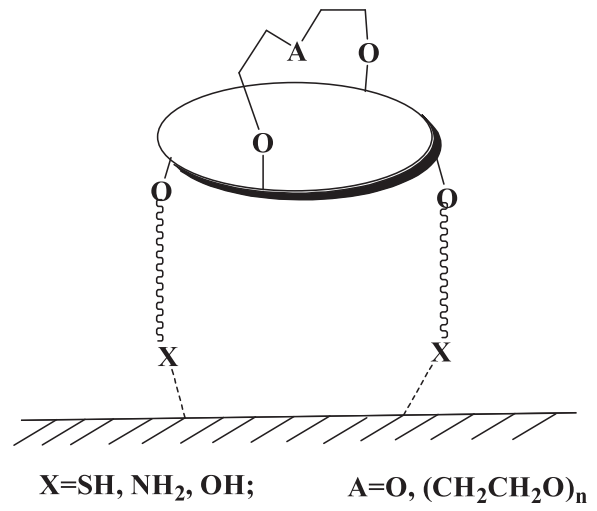

Figure 2. Schematic representation of thiacalix[4]monocrowns immobilized on a solid substrate.

This work is devoted to the synthesis of $\omega$-bromofunctionalized thiacalix-monocrowns in 1,3-alternate conformation. Terminal C-Br bonds can be easily undergone further transformations to achieve the optimal interactions of receptor with an investigated surface. It was also important to establish if the size of terminal substituents will have an effect on the complexation characteristics of crown ether fragment, because calixarenes are the allosteric systems. 


\section{Experimental}

Solvents were purified according to the known procedures. [19] Parent thiacalix[4]arene 3 was synthesized according to the procedure described earlier. ${ }^{[20]}$ Oligoethylene glycols with the content of main substance of more than 99\% (diethylene glycol of an Acros Company; triethylene glycol, Acros; and tetraethylene glycol, Alfa Aesar), triphenylphosphine (Acros, 99\%), diethyl azodicarboxylate (Lancaster, 97\%) were used without further purification.

IR spectra of the compounds synthesized were recorded on a Bruker Vector-22 Fourier spectrometer in the wavenumber range from 400 to $4000 \mathrm{~cm}^{-1}$. Melting points of substances were determined on a BOETIUS compact heating table. Mass spectra were recorded on a MALDI-TOF Ultraflex III mass spectrometer from the solutions of substances in appropriate solvent in the concentration range from $10^{-3}$ to $10^{-5} \mathrm{~mol} / 1$ (matrices are $\mathrm{p}$-nitroaniline and dihydroxybenzoic acid). NMR experiments were performed on Avance- 400 and 600 (Bruker) spectrometers in $\mathrm{CDCl}_{3}$ at the temperature of $30^{\circ} \mathrm{C}$ and chemical shifts were determined relatively to the signals of residual protons of deuterated solvents $\left(\mathrm{CDCl}_{3}\right)$. TLC was performed on Silufol UV 254 plates. All reactions were conducted in argon atmosphere.

Compounds $\mathbf{4 a}, \mathbf{b}$ were synthesized according to a procedure previously described. ${ }^{[21]}$ Constants of $\mathbf{4 a}$ are consistent with earlier published data. ${ }^{[21]}$

5,11,17,23-Tetra-tert-butyl-25,27(3-bromopropoxy)-26,28dihydroxy-2,8,14,20-tetrathiacalix[4]arene (4b). 82\%, mp 295$298^{\circ} \mathrm{C}, \mathrm{R}_{\mathrm{f}}($ Hex:EtOAc $=9: 1)$ 0.54. Found: C 57.20, H $5.76 \%$. $\mathrm{C}_{46} \mathrm{H}_{58} \mathrm{O}_{4} \mathrm{~S}_{4} \mathrm{Br}_{2}$ calculated C 57.37, $\mathrm{H}$ 5.69. $\mathrm{m} / z$ (MALDI TOF) (\%) $961(100)\left[(\mathrm{M})^{+}\right], 984(45 \%)\left[(\mathrm{M}+\mathrm{Na})^{+}\right], 1000(10 \%)\left[(\mathrm{M}+\mathrm{K})^{+}\right]$. IR $(\mathrm{KBr}) v_{\max } \mathrm{cm}^{-1}: 3392(\mathrm{OH}) .{ }^{1} \mathrm{H}$ NMR $\left(\mathrm{CDCl}_{3}, 303 \mathrm{~K}\right) \delta_{\mathrm{H}}$ ppm: 7.78 $(2 \mathrm{H}, \mathrm{s}, \mathrm{OH}), 7.66\left(4 \mathrm{H}, \mathrm{s}, \mathrm{H}_{\text {arom }}\right), 7.01\left(4 \mathrm{H}, \mathrm{s}, \mathrm{H}_{\text {arom }}\right), 4.59(4 \mathrm{H}, \mathrm{t}$ $\left.J=4.50 \mathrm{~Hz}, \mathrm{OCH}_{2}\right), 3.83\left(4 \mathrm{H}, \mathrm{t} J=4.50 \mathrm{~Hz}, \mathrm{CH}_{2} \mathrm{Br}\right), 2.58(4 \mathrm{H}, \mathrm{m}$, $\left.\mathrm{CH}_{2} \mathrm{CH}_{2} \mathrm{CH}_{2}\right), 1.34\left(18 \mathrm{H}, \mathrm{s}, \mathrm{C}\left(\mathrm{CH}_{3}\right)_{3}\right), 0.83\left(18 \mathrm{H}, \mathrm{s}, \mathrm{C}\left(\mathrm{CH}_{3}\right)_{3}\right)$.

\section{General Procedure for Synthesis of the Compounds 5-7 and 8-9}

Diethyl azodicarboxylate in toluene solution was added dropwise to the suspension of bromides $\mathbf{4}$, triphenylphosphine and oligoethylene glycols in toluene at room temperature. Reaction conditions and reagents ratio are given in Table 1. The reaction mixture gradually became transparent and toluene was removed under reduced pressure. Addition of methanol gave white precipitate. After column chromatography (eluent is hexane:ethyl acetate from 9:1 to 2:1) individual products were obtained as white powders.

5,11,17,23-Tetra-tert-butyl-25,27-di(2-bromoethoxy)2,8,14,20-tetrathiacalix [4]arene-monocrown-3 (5a). 43\%, mp $310-315^{\circ} \mathrm{C}, \mathrm{R}_{\mathrm{f}}($ Hex:EtOAc $=8: 1)$ 0.70. Found: C 57.85, H 6.37
\%. $\mathrm{C}_{48} \mathrm{H}_{60} \mathrm{O}_{5} \mathrm{~S}_{4} \mathrm{Br}_{2} \cdot 2\left(\mathrm{CH}_{3}\right)_{2} \mathrm{C}(\mathrm{O})$ calculated $\mathrm{C} 57.85, \mathrm{H}$ 6.47. $\mathrm{m} / \mathrm{z}$ (MALDI TOF) (\%) $1027(100)\left[(\mathrm{M}+\mathrm{Na})^{+}\right] . \delta_{\mathrm{H}}\left(\mathrm{CDCl}_{3}, 303 \mathrm{~K}\right) 7.46$ $\left(4 \mathrm{H}, \mathrm{s}, \mathrm{H}_{\text {arom }}\right), 7.31\left(4 \mathrm{H}, \mathrm{s}, \mathrm{H}_{\text {arom }}\right), 4.00\left(4 \mathrm{H}, \mathrm{t} J=8.22 \mathrm{~Hz}, \mathrm{CH}_{2} \mathrm{Br}\right)$, $3.56\left(4 \mathrm{H}, \mathrm{t} J=5.68 \mathrm{~Hz}, \mathrm{OCH}_{2} \mathrm{CH}_{2} \mathrm{O}\right), 2.78(4 \mathrm{H}, \mathrm{t} J=5.52 \mathrm{~Hz}$, $\left.\mathrm{OCH}_{2} \mathrm{CH}_{2} \mathrm{O}\right), 2.52\left(4 \mathrm{H}, \mathrm{t} J=8.16 \mathrm{~Hz}, \mathrm{OCH}_{2} \mathrm{CH}_{2} \mathrm{Br}\right), 1.32(18 \mathrm{H}, \mathrm{s}$, $\left.\mathrm{C}\left(\mathrm{CH}_{3}\right)_{3}\right), 1.29\left(18 \mathrm{H}, \mathrm{s}, \mathrm{C}\left(\mathrm{CH}_{3}\right)_{3}\right)$.

5,11,17,23-Tetra-tert-butyl-25,27-di(3-bromopropoxy)2,8,14,20-tetrathiacalix[4]arene-monocrown-3 (5b). 46\%, mp $273^{\circ} \mathrm{C}, \mathrm{R}_{\mathrm{f}}$ (Hex:EtOAc $=9: 1$ ) 0.59. Found: C 58.15, H $6.21 \%$. $\mathrm{C}_{50} \mathrm{H}_{64} \mathrm{Br}_{2} \mathrm{O}_{5} \mathrm{~S}_{4}$ calculated C 58.13, H 6.24. $\mathrm{m} / z$ (MALDI TOF) (\%) $1033(100)\left[(\mathrm{M})^{+}\right], 1072(100)\left[(\mathrm{M}+\mathrm{K})^{+}\right] .{ }^{1} \mathrm{H}$ NMR $\left(\mathrm{CDCl}_{3}, 303 \mathrm{~K}\right)$ $\delta_{\mathrm{H}}$ ppm: $7.35\left(4 \mathrm{H}, \mathrm{s}, \mathrm{H}_{\text {arom }}\right), 7.33\left(4 \mathrm{H}, \mathrm{s}, \mathrm{H}_{\text {arom }}\right), 3.97(4 \mathrm{H}, \mathrm{t} J=6.42$ $\left.\mathrm{Hz}, \mathrm{CH}_{2} \mathrm{Br}\right), 3.62\left(4 \mathrm{H}, \mathrm{t} J=4.94 \mathrm{~Hz}, \mathrm{OCH}_{2} \mathrm{CH}_{2} \mathrm{O}\right), 3.07(4 \mathrm{H}, \mathrm{t}$ $\left.J=7.02 \mathrm{~Hz}, \mathrm{OCH}_{2} \mathrm{CH}_{2} \mathrm{Br}\right), 2.73\left(4 \mathrm{H}, \mathrm{t} J=5.00 \mathrm{~Hz}, \mathrm{OCH}_{2} \mathrm{CH}_{2} \mathrm{O}\right)$, $1.58\left(4 \mathrm{H}, \mathrm{m} \mathrm{J}=6.74 \mathrm{~Hz}, \mathrm{CH}_{2} \mathrm{CH}_{2} \mathrm{CH}_{2}\right), 1.32\left(18 \mathrm{H}, \mathrm{s}, \mathrm{C}^{2}\left(\mathrm{CH}_{3}\right)_{3}\right)$, $1.29\left(18 \mathrm{H}, \mathrm{s}, \mathrm{C}\left(\mathrm{CH}_{3}\right)_{3}\right)$.

5,11,17,23-Tetra-tert-butyl-25,27-di(2-bromoethoxy)2,8,14,20-tetrathiacalix [4] arene-monocrown-4 (6a). $60 \%, \mathrm{mp} 331^{\circ} \mathrm{C}$ (dec.), $\mathrm{R}_{\mathrm{f}}$ (Hex:EtOAc=9:1) 0.57. $\mathrm{m} / \mathrm{z}$ (MALDI TOF) (\%) 968 (100) $\left[(\mathrm{M}-\mathrm{Br})^{+}\right], 1069\left[(\mathrm{M}+\mathrm{Na})^{+}\right] .{ }^{1} \mathrm{H} \mathrm{NMR}\left(\mathrm{CDCl}_{3}, 303 \mathrm{~K}\right) \delta_{\mathrm{H}} \mathrm{ppm}: 7.46$ (4 H, s, $\left.\mathrm{H}_{\text {arom }}\right), 7.33\left(4 \mathrm{H}, \mathrm{s}, \mathrm{H}_{\text {arom }}\right), 4.05(8 \mathrm{H}, \mathrm{m}), 3.52(4 \mathrm{H}, \mathrm{t} J=3.82$ $\mathrm{Hz}), 2.47(8 \mathrm{H}, \mathrm{m}), 1.35\left(18 \mathrm{H}, \mathrm{s}, \mathrm{C}\left(\mathrm{CH}_{3}\right)_{3}\right), 1.33\left(18 \mathrm{H}, \mathrm{s}, \mathrm{C}\left(\mathrm{CH}_{3}\right)_{3}\right)$.

5,11,17,23-Tetra-tert-butyl-25,27-di(3-bromopropoxy)2,8,14,20-tetrathiacalix[4]arene-monocrown-4 (6b). 53\%, mp $323^{\circ} \mathrm{C}$ (dec.), $\mathrm{R}_{\mathrm{f}}(\mathrm{Hex}:$ EtOAc $=8: 1)$ 0.57. $\mathrm{m} / z($ MALDI TOF) $(\%)$ $1097(100)\left[(\mathrm{M}+\mathrm{Na})^{+}\right] .{ }^{1} \mathrm{H} \mathrm{NMR}\left(\mathrm{CDCl}_{3}, 303 \mathrm{~K}\right) \delta_{\mathrm{H}} \mathrm{ppm}: 7.40(4 \mathrm{H}$, s, $\left.\mathrm{H}_{\text {arom }}\right), 7.35\left(4 \mathrm{H}, \mathrm{s}, \mathrm{H}_{\text {arom }}\right), 3.98(8 \mathrm{H}, \mathrm{s}), 3.46(4 \mathrm{H}, \mathrm{s}), 3.13(4 \mathrm{H}$, s), $2.54(4 \mathrm{H}, \mathrm{s}), 1.32(40 \mathrm{H}, \mathrm{s})$.

5,11,17,23-Tetra-tert-butyl-25,27-di(2-bromoethoxy)2,8,14,20-tetrathiacalix[4]arene-monocrown-5 (7a). 36\%, mp $310^{\circ} \mathrm{C}, \mathrm{R}_{\mathrm{f}}(\mathrm{Hex}: \mathrm{EtOAc}=4: 1)$ 0.45. Found: C 57.85, H $6.37 \%$. $\mathrm{C}_{52} \mathrm{H}_{68} \mathrm{Br}_{2} \mathrm{O}_{7} \mathrm{~S}_{4} \cdot 2\left(\mathrm{CH}_{3}\right)_{2} \mathrm{C}(\mathrm{O})$ calculated $\mathrm{C}$ 57.85, H 6.47. $\mathrm{m} / \mathrm{z}$ (MALDI TOF) (\%) $1092(100)\left[(\mathrm{M})^{+}\right], 1116\left[(\mathrm{M}+\mathrm{Na})^{+}\right], 1132$ $\left[(\mathrm{M}+\mathrm{K})^{+}\right] .{ }^{1} \mathrm{H} \mathrm{NMR}\left(\mathrm{CDCl}_{3}, 303 \mathrm{~K}\right) \delta_{\mathrm{H}} \mathrm{ppm}: 7.36\left(4 \mathrm{H}, \mathrm{s}, \mathrm{H}_{\text {arom }}\right)$, $7.34\left(4 \mathrm{H}, \mathrm{s}, \mathrm{H}_{\text {arom }}\right), 4.02\left(4 \mathrm{H}, \mathrm{t} J=8.24 \mathrm{~Hz}, \mathrm{CH}_{2} \mathrm{Br}\right), 3.94(4 \mathrm{H}, \mathrm{t}$ $J=9.14 \mathrm{~Hz}$ ), $3.57(4 \mathrm{H}, \mathrm{t} J=2.96 \mathrm{~Hz}), 3.37(4 \mathrm{H}, \mathrm{t} J=3.24 \mathrm{~Hz})$, $3.01(4 \mathrm{H}, \mathrm{t} J=7.90 \mathrm{~Hz}), 2.42\left(4 \mathrm{H}, \mathrm{t} J=8.20 \mathrm{~Hz}, \mathrm{OCH}_{2} \mathrm{CH}_{2} \mathrm{Br}\right)$, $1.35\left(18 \mathrm{H}, \mathrm{s}, \mathrm{C}\left(\mathrm{CH}_{3}\right)_{3}\right), 1.33\left(18 \mathrm{H}, \mathrm{s}, \mathrm{C}\left(\mathrm{CH}_{3}\right)_{3}\right)$.

5,11,17,23-Tetra-tert-butyl-25,27-di(3-bromopropoxy)2,8,14,20-tetrathiacalix [4]arene-monocrown-5 (7b). 23\%, mp $313^{\circ} \mathrm{C}, \mathrm{R}_{\mathrm{f}}$ (Hex:EtOAc $=9: 1$ ) 0.36. Found: C 56.87, H 7.30\%. $\mathrm{C}_{54} \mathrm{H}_{72} \mathrm{Br}_{2} \mathrm{O}_{7} \mathrm{~S}_{4} \cdot 5$ EtOAc calculated C 56.91, H 7.23. $\mathrm{m} / z$ (MALDI TOF) $(\%) 925(100)\left[(\mathrm{M}-\mathrm{Br})^{+}\right], 1006(100)\left[(\mathrm{M}-\mathrm{TTEG}+2 \mathrm{Na})^{+}\right]$, $1024(100)\left[(\mathrm{M}-\mathrm{TTEG}+\mathrm{Na}+\mathrm{K})^{+}\right], 1142(100)\left[(\mathrm{M}+\mathrm{Na})^{+}\right], 1158(100)$ $\left[(\mathrm{M}+\mathrm{K})^{+}\right] .{ }^{1} \mathrm{H} \mathrm{NMR}\left(\mathrm{CDCl}_{3}, 303 \mathrm{~K}\right) \delta_{\mathrm{H}} \mathrm{ppm}: 7.38\left(4 \mathrm{H}, \mathrm{s}, \mathrm{H}_{\text {arom }}\right), 7.31$ $\left(4 \mathrm{H}, \mathrm{s}, \mathrm{H}_{\text {arom }}\right), 3.96\left(4 \mathrm{H}, \mathrm{t} J=6.70 \mathrm{~Hz}, \mathrm{CH}_{2} \mathrm{Br}\right), 3.89(4 \mathrm{H}, \mathrm{t} J=8.20$ $\mathrm{Hz}), 3.61(4 \mathrm{H}, \mathrm{t} J=3.64 \mathrm{~Hz}), 3.38(4 \mathrm{H}, \mathrm{t} J=3.96 \mathrm{~Hz}), 3.05(4 \mathrm{H}$, t $J=6.42 \mathrm{~Hz}), 2.95(4 \mathrm{H}, \mathrm{t} J=8.16 \mathrm{~Hz}), 1.52(4 \mathrm{H}, \mathrm{m} J=6.90 \mathrm{~Hz}$, $\left.\mathrm{CH}_{2} \mathrm{CH}_{2} \mathrm{CH}_{2}\right), 1.36\left(18 \mathrm{H}, \mathrm{s}, \mathrm{C}\left(\mathrm{CH}_{3}\right)_{3}\right), 1.31\left(18 \mathrm{H}, \mathrm{s}, \mathrm{C}\left(\mathrm{CH}_{3}\right)_{3}\right)$.

Table 1. Ratio of yields of monocrowns 5-7 and open-chain derivatives 8-9 under various conditions.

\begin{tabular}{|c|c|c|c|c|c|c|}
\hline & & Temperature, ${ }^{\circ} \mathrm{C}$ & Time, $\mathrm{h}$ & $\begin{array}{c}\text { Reagents ratio } \\
\text { Glycol:(TPP/DEAD):4 ratio }\end{array}$ & Yield 5-7, \% & Yield 8-9, \% \\
\hline \multirow{5}{*}{$\mathrm{n}=1$} & \multirow{2}{*}{$\mathrm{m}=1$} & 20 & 1.5 & $1.5: 3: 1$ & $43(5 a)$ & $27(\mathbf{8 a})$ \\
\hline & & 20 & 24 & $1.5: 3: 1$ & $25(\mathbf{5 a})$ & $25(8 \mathbf{a})$ \\
\hline & $\mathrm{m}=2$ & 20 & 2 & $2: 4: 1$ & $60(6 a)$ & - \\
\hline & \multirow{2}{*}{$\mathrm{m}=3$} & 20 & 3 & $1.5: 3: 1$ & $32(7 \mathbf{a})$ & $28(9 a)$ \\
\hline & & 20 & 3 & $3: 6: 1$ & $36(7 a)$ & $32(9 a)$ \\
\hline \multirow{6}{*}{$\mathrm{n}=2$} & \multirow{3}{*}{$\mathrm{m}=1$} & 20 & 2 & $3: 6: 1$ & $46(5 b)$ & $10(\mathbf{8 b})$ \\
\hline & & 20 & 48 & $3: 6: 1$ & $27(\mathbf{5 b})$ & $32(\mathbf{8 b})$ \\
\hline & & 80 & 2 & $1.5: 3: 1$ & $10(\mathbf{5 b})$ & - \\
\hline & $\mathrm{m}=2$ & 20 & 2 & $2: 4: 1$ & $61(\mathbf{6 b})$ & - \\
\hline & \multirow{2}{*}{$\mathrm{m}=3$} & 20 & 2 & $2: 4: 1$ & $26(7 \mathbf{b})$ & $10(9 b)$ \\
\hline & & 20 & 2 & $3: 6: 1$ & $23(7 \mathbf{b})$ & - \\
\hline
\end{tabular}


5,11,17,23-Tetra-tert-butyl-25,27-di(2-bromoethoxy)-26,28di(5-hydroxy-3-oxopentyl)-2,8,14,20-tetrathiacalix[4]arene (8a). $32 \%, \mathrm{mp} 269^{\circ} \mathrm{C}, \mathrm{R}_{\mathrm{f}}(\mathrm{Hex}:$ EtOAc $=2: 1)$ 0.06. Found: C 48.13, H 5.40 $\% . \mathrm{C}_{52} \mathrm{H}_{70} \mathrm{Br}_{2} \mathrm{O}_{5} \mathrm{~S}_{4} \cdot 2 \mathrm{CHCl}_{3}$ calculated $\mathrm{C} 48.05, \mathrm{H}$ 5.38. $\mathrm{m} / z$ (MALDI TOF) $(\%) 1133(100)\left[(\mathrm{M}+\mathrm{Na})^{+}\right], 1149\left[(\mathrm{M}+\mathrm{K})^{+}\right] . \mathrm{IR}(\mathrm{KBr}) v_{\max }$ $\mathrm{cm}^{-1}: 3484(\mathrm{OH}) .{ }^{1} \mathrm{H}$ NMR $\left(\mathrm{CDCl}_{3}, 303 \mathrm{~K}\right) \delta_{\mathrm{H}} \mathrm{ppm}: 7.40(4 \mathrm{H}, \mathrm{s}$, $\left.\mathrm{H}_{\text {arom }}\right), 7.35\left(4 \mathrm{H}, \mathrm{s}, \mathrm{H}_{\text {arom }}\right), 4.07\left(4 \mathrm{H}, \mathrm{t} J=7.46 \mathrm{~Hz}, \mathrm{CH}_{2} \mathrm{Br}\right), 4.04$ (4 $\mathrm{H}, \mathrm{t} J=5.98 \mathrm{~Hz}), 3.62(4 \mathrm{H}, \mathrm{t} J=3.78 \mathrm{~Hz}), 3.38(4 \mathrm{H}, \mathrm{t} J=4.58 \mathrm{~Hz})$, $3.14(4 \mathrm{H}, \mathrm{t} J=6.88 \mathrm{~Hz}), 2.57\left(4 \mathrm{H}, \mathrm{t} J=8.24 \mathrm{~Hz}, \mathrm{OCH}_{2} \mathrm{CH}_{2} \mathrm{Br}\right)$, $1.32\left(18 \mathrm{H}, \mathrm{s}, \mathrm{C}\left(\mathrm{CH}_{3}\right)_{3}\right), 1.30\left(18 \mathrm{H}, \mathrm{s}, \mathrm{C}\left(\mathrm{CH}_{3}\right)_{3}\right)$.

5,11,17,23-Tetra-tert-butyl-25,27-di(3-bromopropoxy)26,28-di(5-hydroxy-3-oxopentyl)-2,8,14,20-tetrathiacalix [4]arene (8b). $27 \%, \mathrm{mp} 275-280^{\circ} \mathrm{C}, \mathrm{R}_{\mathrm{f}}(\mathrm{Hex}:$ EtOAc $=3: 1)$ 0.15. Found: $\mathrm{C}$ 55.96, $\mathrm{H}$ 6.56, $\mathrm{S} 11.12 \%$. $\mathrm{C}_{50} \mathrm{H}_{64} \mathrm{Br}_{2} \mathrm{O}_{5} \mathrm{~S}_{4} \cdot \mathrm{H}_{2} \mathrm{O}$ calculated $\mathrm{C} 56.05$, H 6.62, S 11.08. $\mathrm{m} / z$ (MALDI TOF) (\%) 1138 (100) [(M) $], 1162$ $\left[(\mathrm{M}+\mathrm{Na})^{+}\right], 1178\left[(\mathrm{M}+\mathrm{K})^{+}\right] .{ }^{1} \mathrm{H}$ NMR $\left(\mathrm{CDCl}_{3}, 303 \mathrm{~K}\right) \delta_{\mathrm{H}}$ ppm: 7.40 (4 H, s, $\left.\mathrm{H}_{\text {arom }}\right), 7.34\left(4 \mathrm{H}, \mathrm{s}, \mathrm{H}_{\text {arom }}\right), 4.01\left(8 \mathrm{H}\right.$, br. s., $\left.\mathrm{CH}_{2} \mathrm{Br}\right), 3.65$ $(4 \mathrm{H}$, br. s.), $3.41(4 \mathrm{H}$, br. s.), $3.09(4 \mathrm{H}, \mathrm{t} J=6.70 \mathrm{~Hz}), 3.03(4 \mathrm{H}$, t $J=6.98 \mathrm{~Hz}), 1.65(4 \mathrm{H}, \mathrm{t} J=6.58 \mathrm{~Hz}), 1.31\left(18 \mathrm{H}, \mathrm{s}, \mathrm{C}\left(\mathrm{CH}_{3}\right)_{3}\right)$, $1.31\left(18 \mathrm{H}, \mathrm{s}, \mathrm{C}\left(\mathrm{CH}_{3}\right)_{3}\right)$.

5,11,17,23-Tetra-tert-butyl-25,27-di(2-bromoethoxy)-26,28di(9-hydroxy-3,5,7-trioxopentyl)-2,8,14,20-tetrathiacalix [4]arene (9a). 32\%, mp 202 ${ }^{\circ} \mathrm{C}, \mathrm{R}_{\mathrm{f}}$ (Hex:EtOAc $\left.=2: 1\right)$ 0.10. $\mathrm{m} / \mathrm{z}$ (MALDI TOF) (\%) $1287(100)\left[(\mathrm{M})^{+}\right], 1310\left[(\mathrm{M}+\mathrm{Na})^{+}\right], 1327\left[(\mathrm{M}+\mathrm{K})^{+}\right]$, $1367\left[(\mathrm{M}+2 \mathrm{~K})^{+}\right]$. IR $(\mathrm{KBr}) v_{\max } \mathrm{cm}^{-1}: 3484(\mathrm{OH}) .{ }^{1} \mathrm{H} \mathrm{NMR}\left(\mathrm{CDCl}_{3}\right.$, $303 \mathrm{~K}) \delta_{\mathrm{H}}$ ppm: $7.36\left(4 \mathrm{H}, \mathrm{s}, \mathrm{H}_{\text {arom }}\right), 7.35\left(4 \mathrm{H}, \mathrm{s}, \mathrm{H}_{\text {arom }}\right), 4.28(4 \mathrm{H}$, br. s.), 4.18 (4 H, br. s.), 4.06 ( $8 \mathrm{H}$, br. s.), 3.71 (4 H, br. s.), 3.57 (8 H, br. s.), 3.44 (4 H, br. s.), 3.03 (4 H, t $J=6.20 \mathrm{~Hz}$ ), 2.59 (4 H, br. s.), $1.30\left(36 \mathrm{H}\right.$, br. s., $\left.\mathrm{C}\left(\mathrm{CH}_{3}\right)_{3}\right)$.

5,11,17,23-Tetra-tert-butyl-25,27di(3-bromopropoxy)-26,28di(9-hydroxy-3,5,7-trioxopentyl)-2,8,14,20-tetrathiacalix[4]arene (9b). 10\%, $\mathrm{R}_{\mathrm{f}}$ (Hex:EtOAc $\left.=2: 1\right)$ 0.05. Found: C 56.60, H 7.01 $\% . \mathrm{C}_{62} \mathrm{H}_{90} \mathrm{Br}_{2} \mathrm{O}_{12} \mathrm{~S}_{4}$ calculated C 56.61, H 6.90. m/z (MALDI TOF) (\%) $1160(100)\left[(\mathrm{M}-\mathrm{TTEG}+\mathrm{Na})^{+}\right]$. IR (KBr) $v_{\max } \mathrm{cm}^{-1}: 3484(\mathrm{OH})$. ${ }^{1} \mathrm{H} \mathrm{NMR}\left(\mathrm{CDCl}_{3}, 303 \mathrm{~K}\right) \delta_{\mathrm{H}} \mathrm{ppm}: 7.40\left(4 \mathrm{H}, \mathrm{s}, \mathrm{H}_{\text {arom }}\right), 7.33(4 \mathrm{H}, \mathrm{s}$, $\left.\mathrm{H}_{\text {arom }}\right), 4.28$ (4 H, br. s.), 4.17 (4 H, br. s.), 4.01 (8 H, br. s.), 3.67 (8 H, br. s.), 3.57 (4 H, br. s.), 3.46 (4 H, br. s.), 3.08 (4 H, t $J=6.20$ $\mathrm{Hz}), 2.98$ (4 H, br. s.), $1.67(4 \mathrm{H}, \mathrm{m}), 1.31\left(36 \mathrm{H}\right.$, br. s, $\left.\mathrm{C}\left(\mathrm{CH}_{3}\right)_{3}\right)$.

\section{Procedure of Extraction Studies}

Alkali metal picrates were obtained by mixing aqueous solutions of picric acid and metal hydroxide solutions, which were preliminarily titrated by $0.1 \mathrm{M} \mathrm{HCl}$. Aqueous solutions of picrates (4 $\mathrm{ml}$ ), which contain excess metal hydroxide, and the solutions of the compounds under study in $\mathrm{CH}_{2} \mathrm{Cl}_{2}(4 \mathrm{ml})$ were stirred for $60 \mathrm{~min}$ at $\sim 20^{\circ} \mathrm{C}$ and kept for $90 \mathrm{~min}$ for phase separation. Initial concentrations of ligand in organic phase and metal hydroxide and picrate in aqueous phase corresponded to $[5-7]_{0}=10^{-2} \mathrm{~mol} \cdot \mathrm{l}^{-1},[\mathrm{MOH}]_{0}=10^{-1} \mathrm{~mol} \cdot \mathrm{l}^{-1}$, and $[\mathrm{HPic}]_{0}=5.0 \cdot 10^{-3} \mathrm{~mol} \cdot 1^{-1}$, respectively. Optical densities of aqueous phase prior to and after extraction ( $A_{i}$ and $A_{0}$, respectively) were determined according to UV spectroscopy on a Perkin Elmer Lambda 35 device at $355 \mathrm{~nm}$ Extraction percentage $(\% E)$ was calculated according to the following relationship: $\% E=\left[\left(A_{0}-A_{i}\right) / A_{0}\right] \cdot 100$.

\section{Results and Discussion}

Synthesis of monocrown bis-omega-bromalkoxy thiacalix[4]arenes can be performed according to two pathways (Scheme 1).

The former involves the preliminary formation of crown-ether fragment (Scheme 1, a) on a calixarene platform and further functionalization of remaining two phenolic hydroxyl groups by $\alpha, \omega$-dihaloalkanes. However, in the case of thiacalix[4]arenes, on the first step the formation of some by-products was observed, namely, products of 1,2macrocyclization (Scheme 1, b), biscalixarenes ${ }^{[22]}$ (Scheme $1, \mathbf{c})$, and biscrown-ether derivatives in 1,3- and 1,2-alternate conformations $^{[23]}$ (Scheme 1, d, e) and the target product can be obtained in rather low yield (Scheme 1, g). Other approach represents the primary synthesis of distal disubstituted thiacalixarenes (Scheme 1, f) with further crosslinking of remaining free hydroxyl groups by oligoethylene glycols. The second way looks more preferable because the formation of biscrown-ether derivatives (c and d) as well as 1,2-cyclization products (b and e) is principally blocked (Scheme 1). Moreover the convenient method of synthesis of thiacalix[4]arene distal derivatives in high yield was recently discovered. ${ }^{[21]}$

Starting compounds, namely, distal substituted $\omega$-bromoderivatives of thiacalix[4]arene $\mathbf{4 a}, \mathbf{b}$ with the number of methylene units of 2 and 3 (Scheme 2) were synthesized according to Mitsunobu protocol ${ }^{[21]}$ from corresponding bromo-substituted alcohols. Their spatial structure was elucidated from 1D NOE measurements. NOE's between protons of $\mathrm{CH}_{2}$ and $\mathrm{OH}$ groups of lower rim substituents as well as between tert-butyl protons and aromatic protons of calixarene rim are observed. Keeping in mind the symmetry of NMR spectra, we can conclude that macrocycles $\mathbf{4 a}, \mathbf{b}$ adopt the cone stereoisomeric form in solution.

The formation of crown ether fragment on disubstituted derivatives 4 was performed by their reaction with a series of oligoethylene glycols, namely, di-, tri-, and tetraethylene glycols, underMitsunobuprotocol.Bothtargetthiacalixcrowns 5-7 and their open-chain products $\mathbf{8 , 9}$ were isolated from the reaction mixture by column chromatography (Scheme 3 ). It should be noted that the formation of such open-chain derivatives in thiacalixarene chemistry was not observed earlier. Compounds $\mathbf{8}$ and $\mathbf{9}$ can represent special interest

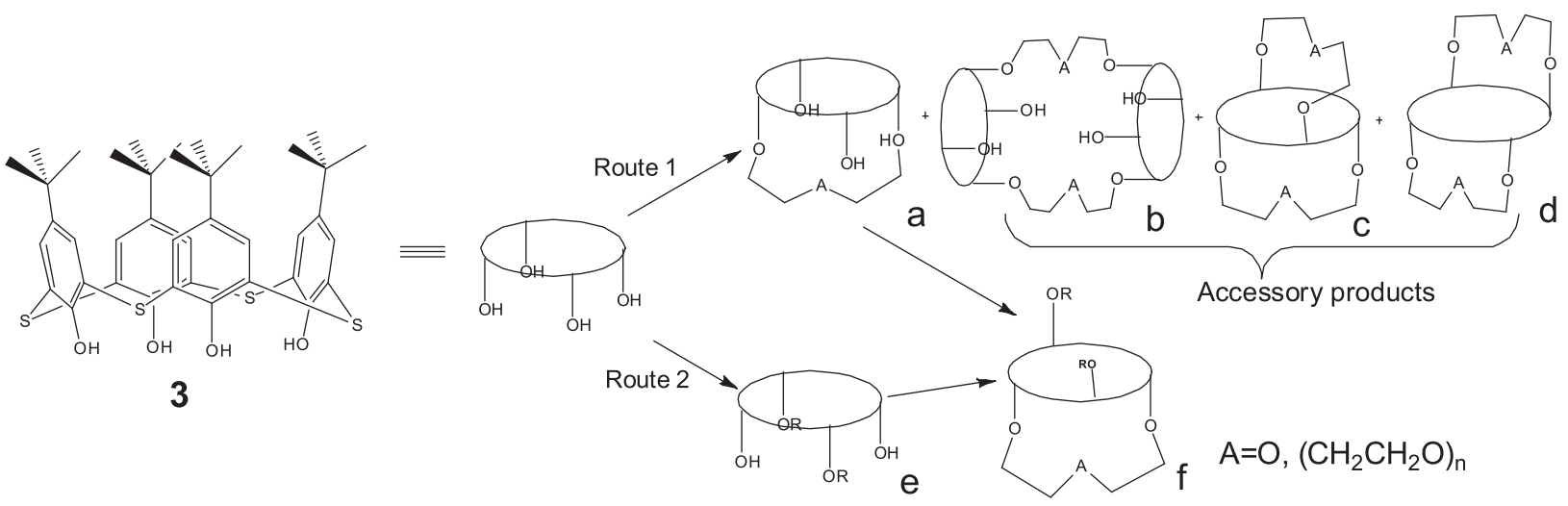

Scheme 1. Possible pathways of the synthesis of distally substituted thiacalix-crowns. 
because terminal $\mathrm{OH}$ and $\mathrm{Br}$ groups located on the different sides of 1,3-alternate give unique possibility to construct sophisticated structures capable of acting as multifunctional devices, for example, sensor systems.

Macrocyclization of distal derivatives has several features. We established the first example of intramolecular crosslinking of distal hydroxyl groups of thiacalix[4]arene by diethylene glycol under Mitsunobu protocol. Usually, unsubstituted thiacalix[4]arene $\mathbf{3}$ gives bis-thiacalix[4]arene $\mathbf{1 0}$ whereas 1,2-crosslinked product $\mathbf{1 1}$ is formed with calix[4] arene in analogous reaction (Scheme 4). ${ }^{[22]}$

To explain such behavior the formation of betaine intermediate $\mathbf{1 2}$ in 1,3-alternate stereoisomeric form has been suggested ${ }^{[24]}$ (Figure 3). In this case, intramolecular crosslinking is more preferential compared to intermolecular interaction due to the steric effects, which are caused by the presence of tert-butyl groups preventing the approach of the second calixarene molecule. At the same time, the reaction with the second glycol molecule should proceed substantially easier and leads to the formation of open-chain podands $\mathbf{8}, \mathbf{9}$.

It is well known the yield of macrocyclization products depends on several factors. The main of them is the special

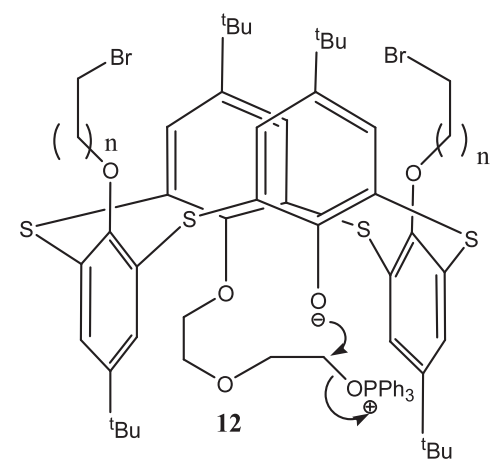

Figure 3. Possible structure of betaine intermediate $\mathbf{1 2}$.

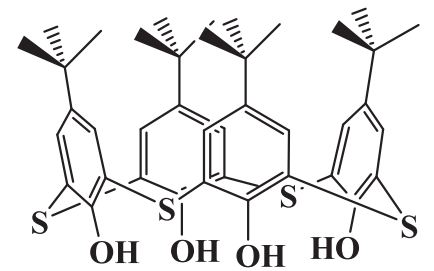

3

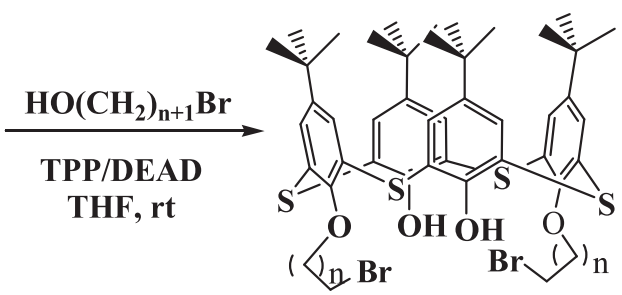

4a $n=183 \%$

4b $n=282 \%$

Scheme 2. Synthesis of dibromoalkoxythiacalixarenes 4 by Mitsunobu reaction.

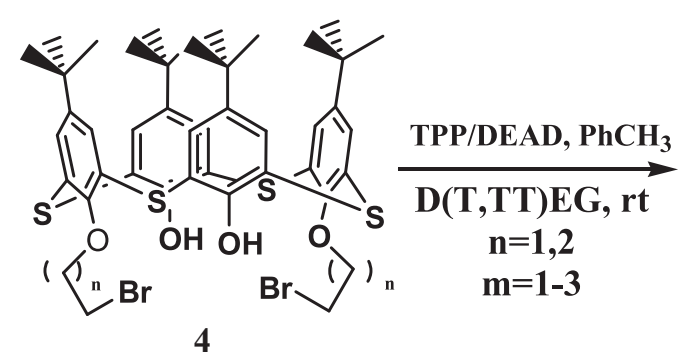

4

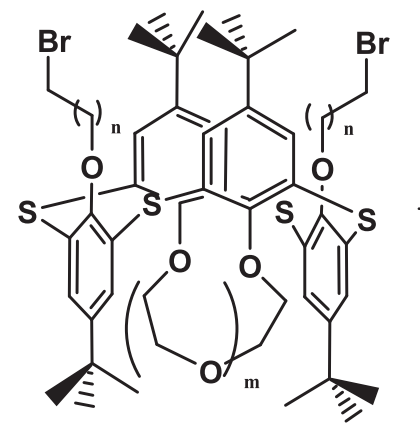

5a: $\mathrm{m}=1, \mathrm{n}=1$

5b: $\mathrm{m}=1, \mathrm{n}=2$

6a: $m=2, n=1$

6b: $m=2, n=2$

7a: $m=3, n=1$

7b: $\mathrm{m}=3, \mathrm{n}=2$

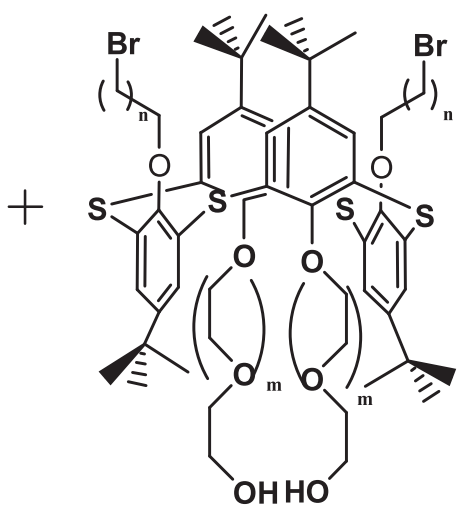

8a: $m=1, n=1$

8b: $m=1, n=2$

9a: $m=3, n=1$

9b: $\mathrm{m}=3, \mathrm{n}=2$

Scheme 3. Reaction of dibromoalkoxythiacalixarenes $\mathbf{4}$ with oligoethylene glycols.

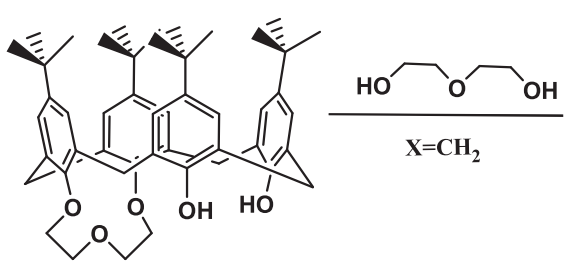

11

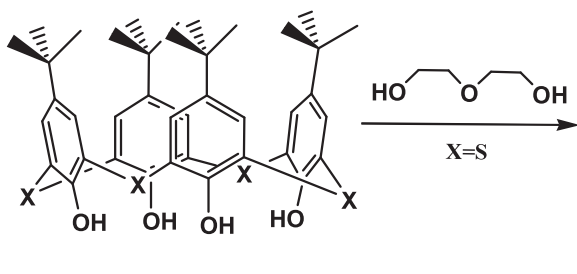

m)

Scheme 4. Reaction of calix[4]arene and thiacalix[4]arene with diethylene glycol. ${ }^{[22]}$

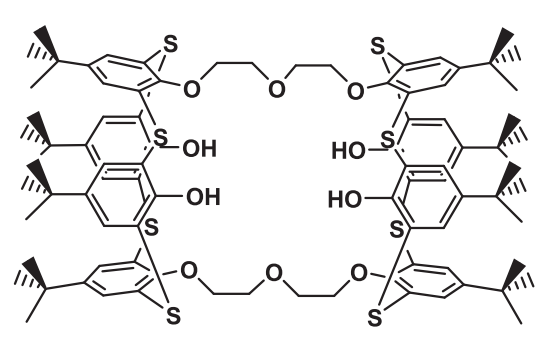

10 
and energetical complementarity of the reacting molecules. In our case, the effectiveness of the crown ether fragment formation should depend on the glycol flexibility (number of oxyethyl fragments in glycols) and thermodynamic stability of the cyclic system formed.

Analysis of Table 1 indicates that the reaction under study is extremely sensitive to the number of oxyethyl fragments in glycols. In the case of triethylene glycol, the formation of only cyclic products was observed in rather good yield ( $c a$. $60 \%$ ) and open-chain products are not observed in the reaction mixture. Involvement in the reaction of shorter or longer glycols leads to the formation of mixture of crown ether and open-chain products. So, triethylene glycol is optimal reagent for macrocyclization of distal substituted thiacalix[4]arenes.

To optimize the conditions of synthesis, an effect of temperature and reaction time on the yields of cyclic and open-chain products was estimated on the example of the reaction of dibromopropoxy derivative $\mathbf{4 b}$ with diethylene glycol (Table 1). The increase of temperature up to $80^{\circ} \mathrm{C}$ leads to decrease of the yields of the both products. It can be caused by competitive reaction of $\mathrm{C}-\mathrm{Br}$ bond and triphenylphosphine being in excess with phosphonium salts formation. Reaction time increasing (from 2 to 48 hours) gives rise to increase the amount of podand in reaction mixture. It can be explained that investigated reaction is an equilibrium and gradually the more thermodynamically stable open-chain product becomes dominant. Thus, macrocyclization is kinetically controlled process, while the formation of open-chain products proceeds under thermodynamic control. Therefore, factors, which increase the rate of reaction, will provide macrocyclization. In particular, large excess of glycol, as well as triphenylphosphinediethyl azodicarboxylate (TPP/DEAD) provides the increase of thiacalixcrowns yield (Table 1).

Structure of compounds $\mathbf{5 a}, \mathbf{b}$ and $\mathbf{8 a}, \mathbf{b}$ was established on the basis of homo- and heteronuclear correlation NMR experiments (1D/2D DEPT, COSY, HSQC, and HMBC). Conclusions on the spatial structure are based on the estimations of NOE (1D DPFGNOE). ${ }^{[25]}{ }^{1} \mathrm{H}$ NMR spectra of the products correspond to the structure with high symmetry, that is, cone or 1,3-alternate stereoisomeric forms. However, the presence NOE between protons of methylene and tertbutyl groups and aromatic protons of thiacalixarene cycle (Figure 4) indicates unambiguously the formation of 1,3alternate stereoisomers.

\section{Complexation Ability of Monocrown-Thiacalixarenes}

To evaluate the complexation ability of the monocrownthiacalix[4]arenes towards alkali metal cations, the liquid extraction of their picrates in a water-dichloromethane system was performed. The results obtained are given in Table 2.

One can see thiacalix-monocrowns $\mathbf{5}$ and $\mathbf{6}$ did not demonstrate substantial binding efficiency and selectivity of alkali metal cations. It is obviously that crown ether cavity of these macrocycles is not suitable for the incorporation of any alkali metal cations. It seems that cavity size is too small for effective binding of these ions. In the case of monocrowns $7 \mathbf{a}, \mathbf{b}$ formed by tetraethylene glycol the drastic changes were observed. They demonstrate not only large extraction ability towards alkali metal cations but also good selectivity relatively potassium and rubidium cations. For

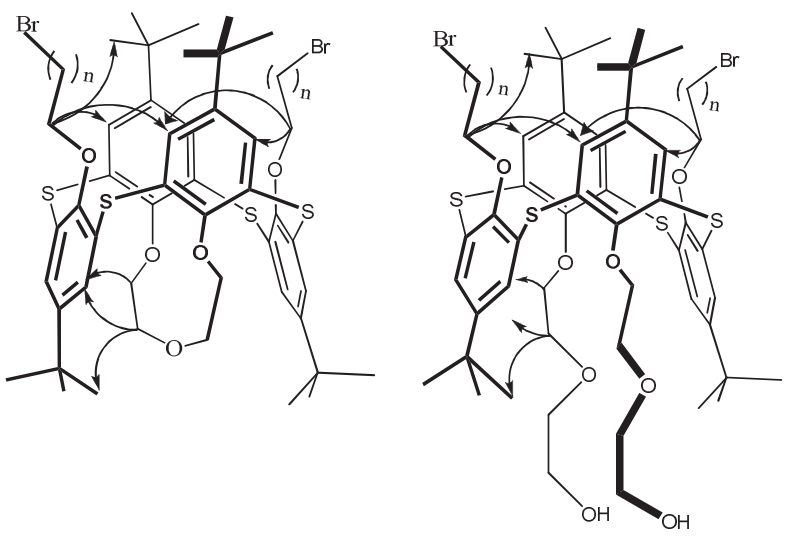

Figure 4. NOE (arrows) in compounds $\mathbf{5 a}, \mathbf{b}$ and $\mathbf{8 a}, \mathbf{b}$

Table 2. Extraction percentage ( $E, \%)$ of alkali metal cations by monocrowns 5-7.

\begin{tabular}{cccccc}
\hline \multirow{2}{*}{ Compounds } & \multicolumn{5}{c}{ Cations } \\
\cline { 2 - 6 } & $\mathrm{Li}$ & $\mathrm{Na}$ & $\mathrm{K}$ & $\mathrm{Rb}$ & $\mathrm{Cs}$ \\
\hline $\mathbf{5 a}$ & 4.9 & 7.9 & 9.1 & 7.7 & 5.6 \\
$\mathbf{5 b}$ & 9.2 & 8.6 & 3.8 & 0 & 14.4 \\
$\mathbf{6 a}$ & 3.6 & 0.2 & 1.1 & 6.4 & 8 \\
$\mathbf{6 b}$ & 2.6 & 0 & 0 & 4.2 & 8 \\
$\mathbf{7 a}$ & 0.7 & 2.9 & 53.5 & 51.4 & 22.6 \\
$\mathbf{7 b}$ & 11.2 & 0 & 49.8 & 44.9 & 23.2 \\
\hline
\end{tabular}

this reason, it was interesting to determine the effect of the nature of lateral substituents on binding properties of crown ether fragment in the compounds studied. It is well known that calixarenes are allosteric systems, where structural (including conformational) changes of macrocyclic cavity from one side may lead to the drastic changes in another one. This feature should be taken into account upon the use of these compounds as sensors on a solid surface. Immobilization on surface can cause the conformational changes in the macrocycle that can lead to the change or even loss of receptor properties.

To estimate this effect the extraction ability of some thiacalixcrowns with different lateral substituents was compared. For comparison, compounds $\mathbf{1 3}$ and $\mathbf{1 4}$ bearing methyl and propyl substituents were chosen; their extraction degrees of alkali metal cations were determined earlier ${ }^{[18]}$ (with an exception of sodium cation) under the same experimental conditions. It was established (Figure 5) that in a row of methyl - propyl - bromoethyl - bromopropyl, despite the changes in the sizes of substituents, no considerable changes in extraction ability were observed. Thus, substituents which are at the opposite sides from the macrocycle plane do not affect each other and no substantial changes should be expected upon immobilization of these compounds on surface.

\section{Conclusions}

Thus, novel $\omega$-bromoalkoxy-thiacalix[4]arenes 5-7 with crown ether fragments and open-chain podands $\mathbf{8 ,} 9$ in 1,3alternate stereoisomeric form were synthesized under Mitsunobu protocol. The formation of open-chain derivatives 8-9 was not known earlier in the chemistry of calixarenes. For 


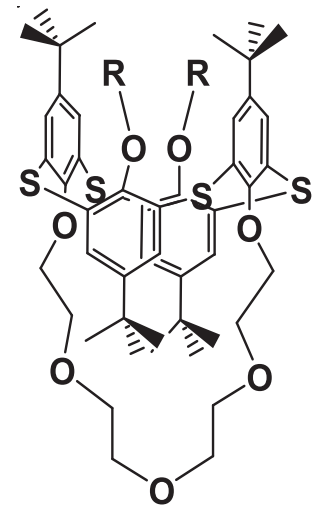

$13 \mathrm{R}=\mathrm{CH}_{3}$ $14 \mathrm{R}=\mathrm{C}_{3} \mathrm{H}_{7}$

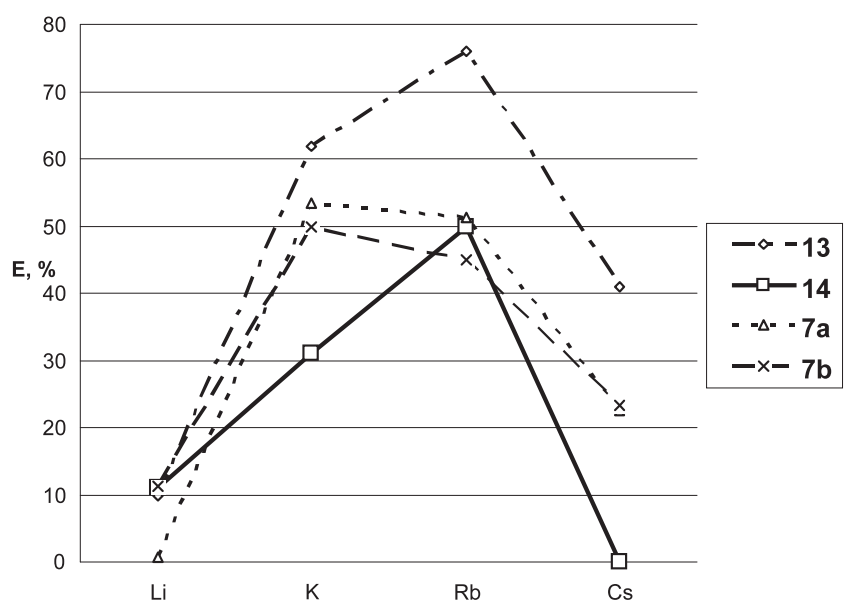

(a)

Figure 5. Alkali metal picrate extraction by monocrowns 7, 13, and 14 .

the first time the intramolecular crosslinking of 1,3-hydroxyl groups of distally substituted thiacalix[4]arenes by the short spacer of diethylene glycol was observed. It was shown that the yields of cyclic 5-7 and open-chain products 8,9 substantially depend on the reaction conditions (temperature, time and reagents ratio). Triethylene glycol is optimal reagent for macrocyclization of distally substituted thiacalix[4]arenes. Involvement in the reaction of shorter or longer glycols leads to the formation of mixture of crown ether and open-chain products.

It was shown by the two-phase extraction method that cavity of monocrowns- 5 has the highest binding ability to rubidium and potassium cations. Monocrown ethers with the smaller cavity size did not demonstrate any high selectivity and efficiency of alkali metal cations extraction.

Acknowledgments. The work was supported by the Russian Foundation for Basic Research (project no. 11-03-00985).

\section{References}

1. Salorinne K., Nissinen M. J. Inclusion Phenom. Macrocyclic Chem. 2008, 61, 11-27.

2. FR Patent 93-4566930419, WO 94FR432 940418, Chem. Abstr. 1995, 122, 239730.

3. Lamare V., Dozol J.-F., Ugozolli F., Casnati A., Ungaro R. Eur. J. Org. Chem. 1998, 8, 1559-1568.

4. Lamare V., Dozol J.-F., Fuangswasdi S., Arnaud-Neu F., Thuéry P., Nierlich M., Asfari Z., Vicens J. J. Chem. Soc., Perkin Trans. 2 1999, 271-284.

5. Stoikov I.I., Omran O.A., Solovieva S.E., Latypov S.K., Enikeev K.M., Gubaidullin A.T., Antipin I.S., Konovalov A.I. Tetrahedron 2003, 59, 1469-1476.

6. Antipin I.S., Solov'eva S.E., Stoikov I.I., Vershinina I.S., Pribylova G.A., Tananaev I.G., Myasoedov B.F. Izv. Akad. Nauk, Ser. Khim. 2004, N. 1, 124-129 (in Russ.).

7. Konovalov A.I., Antipin I.S., Mustafina A.R., Solov'eva S.E., Pod'yachev S.N. Koord. Khim. 2004, 30, 4, 1-20 (in Russ.)

8. Solov'eva S.E., Gruner M., Omran A.O., Gubaidullin A.T.,
Litvinov I.A., Habicher W.D., Antipin I.S., Konovalov A.I. Izv. Akad. Nauk, Ser. Khim. 2005, 9, 2041-2049 (in Russ.).

9. Kozlova M.N., Ferlay S., Solovieva S.E., Antipin I.S., Konovalov A.I., Kyritsakas N., Hosseini M.W. Dalton Trans. 2007, 5126-5131.

10. Solov'eva S.E., Kleshnina S.R., Kozlova M.N., Galiullina L.F., Gubaidullin A.T., Latypov Sh.K., Antipin I.S., Konovalov A.I. Izv. Akad. Nauk, Ser. Khim. 2008, 7, 1448-1456 (in Russ.).

11. Kozlova M.N., Ferlay S., Kyritsakas N., Hosseini M.W., Solovieva S.E., Antipin I.S., Konovalov A.I. Chem. Commun. 2009, 18, 2514-2516.

12. Popova N.N., Morgalyuk V.P., Tananaev I.G., Solov'eva S.E., Popova E.V., Antipin I.S. Izv. Akad. Nauk, Ser. Khim. 2011, N. 1, 167-170 (in Russ.).

13. Mel'nikova N.B., Kochetkov E.N., Solovieva S.E., Popova E.V., Antipin I.S., Bol'shakova A.E., Jiltsova O.E., Konovalov A.I. Izv. Akad. Nauk, Ser. Khim. 2011, N. 9, 23-29 (in Russ.).

14. Lamare V., Dozol J.-F., Thuery P., Nierlich M., Asfari Z., Vicens J. J. Chem. Soc., Perkin Trans. 2 2001, 1920-1926.

15. Csokai V., Grun A., Parlagh G., Bitter I. Tetrahedron Lett. 2002, 43, 4153-4156.

16. Bereczki R., Csokai V., Grun A., Bitter I., Tóth K. Anal. Chim. Acta 2006, 569, 42-49.

17. Perez-Casas C., Rahman S., Begum N., Xi Z., Yamato T. $J$ Inclusion Phenom. Macrocyclic Chem. 2008, 60, 173-185.

18. Csokai V., Grun A., Parlagh G., Bitter I. Tetrahedron Lett. 2002, 43, 7627-7629.

19. Gordon A.J., Ford R.A. A Chemists Companion: A Handbook of Practical Data, Techniques, and References New York: Wiley, 1972. 537 p.

20. Iki N., Kabuto C., Fukushima T., Kumagai H., Takeya H., Miyanari S., Miyashi T., Miyano S. Tetrahedron 2000, 56, 1437-1443.

21. Bitter I., Csokai V. Tetrahedron Lett. 2003, 44, 2261-2265.

22. Csokai V., Grun A., Bitter I. Tetrahedron Lett. 2003, 44, 4681-4684.

23. Van Leeuwen F.W.B., Beijleveld H., Kooijman H., Spek A.L., Verboom W., Reinhoudt D.N. Tetrahedron Lett. 2002, 43, 9675-9678.

24. Csokai V., Bitter I. Supramol. Chem. 2003, 16, 611-619.

25. Stott K., Stonehouse J., Keeler J., Hwang T.-L., Shaka A.J. J. Am. Chem. Soc. 1995, 117, 4199-4200. 